\title{
Comparative analysis of apicoplast genomes of Babesia infective to small ruminants in China
}

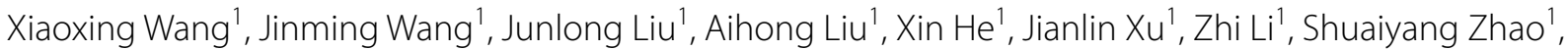 \\ Youquan $\mathrm{Li}^{1}$, Hong Yin ${ }^{1,2}$, Jianxun Luo ${ }^{1}$ and Guiquan Guan ${ }^{1 *}$
}

\begin{abstract}
Background: Babesiosis is an economically important disease caused by tick-borne apicomplexan protists of the genus Babesia. Most apicomplexan parasites, including Babesia, have a plastid-derived organelle termed an apicoplast, which is involved in critical metabolic pathways such as fatty acid, iron-sulphur, haem and isoprenoid biosynthesis. Apicoplast genomic data can provide significant information for understanding and exploring the biological features, taxonomic and evolutionary relationships of apicomplexan parasites, and identify targets for anti-parasitic drugs. However, there are limited data on the apicoplast genomes of Babesia species infective to small ruminants.

Methods: PCR primers were designed based on the previously reported apicoplast genome sequences of Babesia motasi Lintan and Babesia sp. Xinjiang using Illumina technology. The overlapped apicoplast genomic fragments of six ovine Babesia isolates were amplified and sequenced using the Sanger dideoxy chain-termination method. The full-length sequences of the apicoplast genomes were assembled and annotated using bioinformatics software. The gene contents and order of apicoplast genomes obtained in this study were defined and compared with those of other apicomplexan parasites. Phylogenetic trees were constructed on the concatenated amino acid sequences of 13 gene products using MEGA v.6.06.
\end{abstract}

Results: The results showed that the six ovine Babesia apicoplast genomes consisted of circular DNA. The genome sizes were 29,916-30,846 bp with 78.7-81.0\% A + T content, 29-31 open reading frames (ORF) and 23-24 transport RNAs. The ORFs encoded four DNA-directed RNA polymerase subunits (rpoB, rpoCl, rpoC2a and rpoC2b), 13 ribosomal proteins, one elongation factor TU (tufA), two ATP-dependent Clp proteases (ClpC) and 7-11 hypothetical proteins. Babesia sp. has three more genes than Babesia motasi (rp/5, rps8 and rpoB). Phylogenetic analysis showed that Babesia sp. is located in a separate clade. Babesia motasi Lintan/Tianzhu and B. motasi Ningxian/Hebei were divided into two subclades.

Conclusions: To our knowledge, this study is the first to elucidate the whole apicoplast genomic structural features of six Babesia isolates infective to small ruminants in China using Sanger sequencing. The data provide useful information confirming the taxonomic relationships of these parasites and identifying targets for anti-apicomplexan parasite drugs.

Keywords: Babesia motasi, Babesia sp., Apicoplast genome, Assembly and annotation, Comparative analysis

\footnotetext{
*Correspondence: guanguiquan@caas.cn

${ }^{1}$ State Key Laboratory of Veterinary Etiological Biology, Key Laboratory

of Veterinary Parasitology of Gansu Province, Lanzhou Veterinary

Research Institute, Chinese Academy of Agricultural Science, Xujiaping 1,

Lanzhou 730046, Gansu, People's Republic of China

Full list of author information is available at the end of the article
} 


\section{Background}

Most apicomplexan protists cause important diseases of humans and other animals, including malaria (Plasmodium spp.), toxoplasmosis (Toxoplasma gondii), cryptosporidiosis (Cryptosporidium spp.), cyclosporiasis (Cyclospora cayetanensis), coccidiosis (Eimeria spp.), babesiosis (Babesia spp.), theileriosis (Theileria spp.) and neosporosis (Neospora caninum) [1]. These parasites, with the exception of Cryptosporidium spp. [2], possess a unique, essential, vestigial plastid known as the apicoplast. The discovery of the photosynthetic apicomplexan Chromera (chromalveolate hypothesis), the structural characteristics of the apicoplast/plastid genome and phylogenetic analysis of the glyceraldehyde-3-phosphate dehydrogenase and cytochrome $c$ oxidase subunit 2 genes have demonstrated that the apicoplast has evolved through secondary endosymbiosis of a red alga [3-6]. The apicoplast is also involved in critical metabolic pathways such as fatty acid, iron-sulphur, haem and isoprenoid biosynthesis. Previous studies have shown that Plasmodium falciparum, Babesia bovis and Babesia bigemina cannot grow with the antibiotic fosmidomycin that causes loss of the apicoplast $[7,8]$. Interestingly, isopentenyl pyrophosphate supplementation completely reverses death following treatment with fosmidomycin [8]. The apicoplast and some of these metabolic pathways are vital for parasite survival, thus making the apicoplast an attractive target for anti-parasitic drugs.

Babesia spp. are tick-transmitted haemoprotozoa with a worldwide distribution that have been reported to affect domestic animals, wildlife, companion animals and humans [9]. These pathogens cause fever, anaemia, haemoglobinuria and jaundice in acute infections, while chronic infection is asymptomatic. Babesiosis of cattle and small ruminants also has great economic importance due to reduced meat and milk production, and costs associated with treatment, prevention and the disposing of carcasses. The main causative agents of babesiosis in small ruminants are Babesia ovis and Babesia motasi, which are transmitted by Rhipicephalus spp. and Haemaphysalis spp and are distributed in Asia, Africa, South America, Europe and the Far East [9-11]. To date, several ovine Babesia species or geographical isolates, including Babesia sp. Xinjiang (BspXJ), Babesia sp. Dunhuang (BspDH), B. motasi Lintan (BmLT), B. motasi Tianzhu (BmTZ), B. motasi Hebei (BmHB) and B. motasi Ningxian (BmNX), have been isolated from small ruminants in China. They have significant differences in vector specificity, serology, virulence and pathogenicity. Importantly, BspXJ and BspDH are transmitted by Hyalomma anatolicum and mainly cause subclinical infections, whereas BmLT, BmTZ, BmHB and BmNX are transmitted by Haemaphysalis spp. and cause mild to severe clinical signs [12-20]. Seroepidemiological surveys by enzymelinked immunosorbent assay (ELISA) have shown that the prevalence of Babesia sp. and B. motasi are 30.4$31.7 \%$ and $36.0-43.5 \%$, respectively [19, 21-23]. These data indicate that ovine Babesia spp. are widespread in China.

Thus far, sequencing and annotation of the apicoplast genomes have been performed for Plasmodium spp., $T$. gondii, Leucocytozoon caulleryi, Cyclospora cayetanensis, Theileria parva, Eimeria tenella, B. bovis, Babesia microti, Babesia orientalis, BspXJ and BmLT. These studies have revealed that apicoplast genomes lack the genes for metabolic function and regulatory proteins, but are present SNPs and repeats [24-37]. Apicoplast genomic data have been used to understand and explore the biological features of the pathogens, and the taxonomy and evolutionary relationships among apicomplexan parasites [33, 36]. However, there is limited information on the apicoplast genomes of Babesia species infective to small ruminants in China. Although the apicoplast genomes of BspXJ and BmLT have been sequenced and characterized using Illumina technology directly from genomic DNA, they were not verified using PCR amplification and sequencing [36].

In the present study, we employed a Sanger dideoxy chain-termination method to sequence the apicoplast genomes of BspXJ, BspDH, BmLT, BmTZ, BmHB and $\mathrm{BmNX}$, and used bioinformatics software to conduct the assembly and annotation of full sequences of these isolates to verify the published sequences of BspXJ and BmLT sequenced using Illumina technology. Phylogenetic trees were constructed using the apicoplast genomic data to determine the taxonomic relationships among these geographical isolates of Babesia infective to sheep and goats in China. The study provides essential data for clarifying the classification of the Babesia species infective to small ruminants and lays the foundation for performing research on metabolic pathways and identifying diagnostic markers and drug targets for Babesia infections.

\section{Methods}

\section{Parasites and isolation of genomic DNA}

BspXJ, BspDH, BmLT, BmTZ, BmHB and BmNX were isolated from splenectomised sheep that were infected or infested with field-collected sheep blood or ticks from Xinjiang, Hebei and Gansu (Lintan, Tianzhu, Ningxian and Dunhuang counties) provinces during the period 2000-2010. The purified merozoites of BspXJ, BspDH, BmLT, BmTZ, BmHB and BmNX were provided by the vectors and vector-borne diseases laboratory in Lanzhou Veterinary Research Institute, China [38]. Genomic DNA was extracted from the merozoites using a QIAamp DNA 
Blood Mini Kit (Qiagen, Hilden, Germany), according to the manufacturer's instructions. The DNA concentration and quality were measured using the $260 / 280 \mathrm{~nm}$ absorbance ratio on a NanoDrop spectrophotometer (Thermo Fisher Scientific, Waltham, MA, USA). DNA was stored at $-20^{\circ} \mathrm{C}$ until PCR amplification.

\section{Amplification and sequencing of apicoplast genomes}

The PCR primers were designed on the basis of the reported apicoplast genomic sequences of BspXJ (KX881914) and BmLT (KX881915), sequenced using Illumina technology [36]. The primer details are shown in Additional file 1: Table S1. Overlapped fragments covering whole apicoplast genomes were amplified from the genomic DNA of BspXJ, BspDH, BmLT, BmTZ, BmHB and BmNX. The PCR products were directly sequenced using a BigDye Terminator v.3.1 cycle sequencing kit (Applied Biosystems, Foster City, CA, USA) on an ABI 3730 DNA analyser (Applied Biosystems) or cloned into pBluescript II SK(+) using a Clone Express ${ }^{\circledR}$ II One Step Cloning Kit (Vazyme, Nanjing, China) for subsequent sequencing.

\section{Assembly and annotation of the apicoplast genomes}

The CLC Genomics Workbench v.7.5.1 (Qiagen, Redwood City, CA, USA) was used to assemble the apicoplast genomes according to the user manual. Genome annotation was performed using the software Artemis [39] and BLAST (http://blast.ncbi.nlm.nih.gov/Blast.cgi). The use of these two methods guarantees the robustness of the annotation. The E-value of BLAST is 0.0 and we were blasting against a non-redundant protein sequence (nr) database. The tRNA genes were identified using tRNAscan-SE v.2.0 (http://lowelab.ucsc.edu/tRNAscan$\mathrm{SE} /$ ) with the default search mode and other mitochondrial sequence sources [40]. Genetic maps were obtained using the online software CGView (http://stothard.afns. ualberta.ca/cgview_server/) [41]. Mauve (http://gel. ahabs.wisc.edu/mauve) was used to generate the genome comparisons [42]. The nucleotide sequences and annotation information reported in this article were submitted to the GenBank database under the accession numbers MH992224-MH992229.

\section{Phylogenetic analysis}

The 18 apicoplast genomic sequences, six from the ovine Babesia isolates from this study and 12 from apicomplexan parasites obtained from GenBank [Babesia sp. Xinjiang (BspXJ), B. motasi Lintan (BmLT), B. bovis, B. orientalis, $B$. microti, Theileria parva, Plasmodium falciparum, Cyclospora cayetanensis, P. chabaudi chabaudi, Toxoplasma gondii, Eimeria tenella and Leucocytozoon caulleryi], and one chloroplast genomic sequence of
Chromera velie (GenBank: HM222967), were used in the phylogenetic analysis (Table 1). On the basis of the annotation information, 13 encoding genes $(r p l 2, r p l 4, r p l 6$, rpl14, rpl16, rps2, rps3, rps4, rps7, rps11, rps12, tufA and $r o p \mathrm{~B})$ of each genomic sequence were used to deduce their amino acid sequences. The concatenated sequences of 4683 amino acid residues from each species were put into multi-alignment using Clustal W with further manual verification. Subsequently, MEGA v.6.06 (http://www. megasoftware.net/) software was applied to conduct phylogenetic analysis. A bootstrap phylogenetic tree demonstrating the relationship of BspXJ, BspDH, BmLT, BmTZ, $\mathrm{BmNX}$ and $\mathrm{BmHB}$ to other apicomplexan parasites was created by the maximum likelihood (ML) method or neighbour-joining (NJ) method, using a distance matrix corrected for nucleotide substitutions based on the JTT with Freqs model. In addition, phylogeny of the whole apicoplast nucleotide sequence was constructed by the ML method based on the Kimura 2-parameter model. A bootstrap analysis was used to assess the robustness of the clusters using 1000 replicates.

\section{Results \\ Sequence analysis of the apicoplast genomes of ovine Babesia}

Sequencing and assembly revealed that the apicoplast genomes of the six ovine Babesia isolates were formed of circular DNA, ranging from 29,916 to 30,846 bp in length with a high $\mathrm{A}+\mathrm{T}$ content of 78.7-81.0\% (Table 1). Bioinformatics analysis indicated that the circular DNA contained a small subunit and a large subunit ribosomal RNA (SSU and $L S U$ ), 23-24 transfer ribonucleic acids (tRNAs) and five to six ribosomal protein large subunits $(r p l)$, eight to nine ribosomal protein small subunits (rps), four to five subunits of DNA-directed RNA polymerase (rpo), two ATP-dependent Clp proteases $(c l p C 1, c l p C 2)$, one elongation factor $\mathrm{TU}$ (tufA), and seven to eleven hypothetical protein genes (hyp) (Table 2).

All coding genes were transcribed in the same orientation (Additional file 2: Figure S1; Additional file 3: Figure S2; Additional file 4: Figure S3; Additional file 5: Figure S4; Additional file 6: Figure S5; Additional file 7: Figure S6). In total, 7094, 7220, 7384, 7436, 7163 and 7212 amino acids were encoded in the apicoplast genomes of the six ovine Babesia species. All the protein-encoding genes had ATG as a translation start codon. Most of the apicoplast protein-encoding genes had TAA as a translation stop codon, followed by TGA and TAG (Table 3). The alignment of apicoplast genomes of ovine Babesia isolates indicated that the identities of $\mathrm{BspXJ} / \mathrm{DH}$, $\mathrm{BmLT} / \mathrm{TZ}$ and $\mathrm{BmNX} / \mathrm{HB}$ were 99.8 , 99.9 and $99.9 \%$, respectively. BmLT/TZ and $\mathrm{BmNX} / \mathrm{HB}$ had 70.9 and $71.6-71.7 \%$ identity, respectively, to $\mathrm{BspXJ} / \mathrm{DH}$, and that 
Table 1 Comparison of apicoplast genome sequences from 16 apicomplexan parasites

\begin{tabular}{|c|c|c|c|c|c|c|c|c|c|}
\hline Species & Host & GenBank ID & Size (bp) & $\mathrm{A}+\mathrm{T}(\%)$ & $\begin{array}{l}\text { Total no. } \\
\text { of genes }\end{array}$ & $\begin{array}{l}\text { Protein- } \\
\text { encoding } \\
\text { genes }\end{array}$ & rRNA & tRNA & Reference \\
\hline Babesia sp. Xinjiang (BspXJ-Sanger) ${ }^{\text {a1 }}$ & Sheep & MH992224 & 30,758 & 81.0 & 55 & 30 & 2 & 23 & This study \\
\hline Babesia sp. Dunhuang (BspDH) & Sheep & MH992225 & 30,771 & 81.0 & 55 & 30 & 2 & 23 & This study \\
\hline Babesia motasi Lintan (BmLT-Sanger) ${ }^{b 1}$ & Sheep & MH992226 & 30,846 & 78.7 & 57 & 31 & 2 & 24 & This study \\
\hline Babesia motasi Tianzhu (BmTZ) & Sheep & MH992227 & 30,846 & 78.7 & 57 & 31 & 2 & 24 & This study \\
\hline Babesia motasi Ningxian (BmNX) & Sheep & MH992228 & 29,916 & 79.1 & 54 & 29 & 2 & 23 & This study \\
\hline Babesia motasi Hebei (BmHB) & Sheep & MH992229 & 29,921 & 79.1 & 54 & 29 & 2 & 23 & This study \\
\hline Babesia sp. Xinjiang (BspXJ-IIlumina)22 & Sheep & KX881914 & 30,729 & 81.0 & 57 & 30 & 2 & 25 & {$[36]$} \\
\hline Babesia motasi Lintan (BmLT-IIlumina) ${ }^{\mathrm{b} 2}$ & Sheep & KX881915 & 30,738 & 78.7 & 59 & 32 & 2 & 25 & {$[36]$} \\
\hline Babesiabovis & Cattle & NC011395 & 35,107 & 78.0 & 58 & $32^{c}$ & 2 & 24 & {$[28]$} \\
\hline Babesia orientalis & Water buffalo & KT428643 & 33,200 & 79.0 & 64 & 38 & 2 & 24 & [24] \\
\hline Babesia microti & Mouse & LK028575 & 28,657 & 85.9 & 54 & $28^{c}$ & 2 & 24 & {$[25]$} \\
\hline Theileria parva & Cattle & NC007758 & 39,579 & 80.5 & 70 & $44^{c}$ & 2 & 24 & {$[37]$} \\
\hline Plasmodium falciparum & Human & LN999985 & 34,250 & 85.8 & 68 & 30 & 4 & 34 & Unpublished \\
\hline Cyclospora cayetanensis & Human & KP866208 & 34,155 & 78.0 & 65 & 28 & 4 & 33 & {$[44]$} \\
\hline Plasmodium chabaudi chabaudi & Mouse & HF563595 & 29,623 & 86.3 & 59 & 30 & $2^{d}$ & $27^{e}$ & [35] \\
\hline Toxoplasmagondii & Mouse & U87145 & 34,996 & 78.6 & 65 & 28 & 4 & 33 & Unpublished \\
\hline Eimeria tenella & Chicken & AY217738 & 34,750 & 79.4 & 65 & 28 & 4 & 33 & {$[29]$} \\
\hline Leucocytozoon caulleryi & Chicken & AP013071 & 34,779 & 85.1 & 67 & 29 & 4 & 34 & [33] \\
\hline
\end{tabular}

a a1 and a2 are the same sample, sequenced using the Sanger and Illumina method, respectively

${ }^{b}$ b1 and b2 are the same sample, sequenced using the Sanger and Illumina method, respectively

c Two rpoC2 genes were counted as one gene

${ }^{d}$ One large subunit rRNA has a large deletion and was thus removed from the gene count

e One tRNA-R(acg) was removed from the gene count, as it does not have a predicted anticodon

between BmLT/TZ and BmNX/HB was 86.9-87.0\%. Based on the nucleotide sequence analysis of the whole apicoplast, there were multiple base differences among $\mathrm{BspXJ} / \mathrm{DH}, \mathrm{BmLT} / \mathrm{TZ}$ and BmNX/HB (Additional file 8: Table S2).

\section{Alignment of apicoplast genomes with those of other apicomplexan parasites}

Four gene clusters were found in the BspXJ/DH, BmLT/ $\mathrm{TZ}$ and $\mathrm{BmHB} / \mathrm{NX}$ apicoplast genomes. They were in synteny with the same gene clusters of other apicomplexan parasites (Additional file 9: Figure S7). Gene cluster 1 included those encoding ribosomal proteins and tufA genes (Fig. 1). Similar to the gene organization of B. bovis, B. orientalis, B. microti, T. parva, T. gondii, E. tenella and C. cayetanensis apicoplast genomes, the six ovine Babesia isolates lacked the $r p l 23$ gene, but it was present in P. chabaudi chabaudi and P. falciparum between the rpl4 and $r p l 2$ genes. Hypothetical protein genes were not found in Bsp/DH, B. microti, T. parva, T. gondii and $P$. falciparum between rps7 and tufA, whereas BmLT/ $\mathrm{TZ}, \mathrm{BmNX} / \mathrm{HB}, B$. bovis and B. orientalis had two to seven hypothetical protein genes in the locus. Gene cluster 2 consisted of hypothetical protein genes and
$C l p C$ chaperone genes. Similar to B. bovis, B. orientalis, $B$. microti and T. parva, the $C l p C$ genes of the six ovine Babesia isolates were duplicated, with both copies containing the AAA_2 ATPase domain, whereas T. gondii and $P$. falciparum contained one $C l p C$ gene (Fig. 1).

Gene cluster 3 included RpoB, RpoC and rps 2 genes, which were consistent with the gene orientation and contents of cluster 3 in B. bovis, B. orientalis, B. microti and T. parva. Cluster 3 of the piroplasma apicoplast genomes lacks the $s u f \mathrm{~B}$ gene involved in iron-sulphur cluster synthesis. Gene cluster 4 of the piroplasms had several tRNA genes and a single set of SSU and $L S U$ genes, which are transcribed in the same orientation, whereas, this cluster consisted of two sets of $S S U$ and $L S U$ genes in opposite orientations in other parasites. Unlike BspXJ/DH, $\mathrm{BmLT} / \mathrm{TZ}$ and $\mathrm{BmNX} / \mathrm{HB}$, one to two tRNA genes exist between the $S S U$ and $L S U$ genes in B. bovis, B. orientalis, B. microti and T. parva (Fig. 1).

\section{Comparison of genomic data sequenced by the Sanger dideoxy chain-termination method and Illumina technology}

The apicoplast genomic sequences of BspXJ (KX881914) and BmLT (KX881915) sequenced using Illumina 


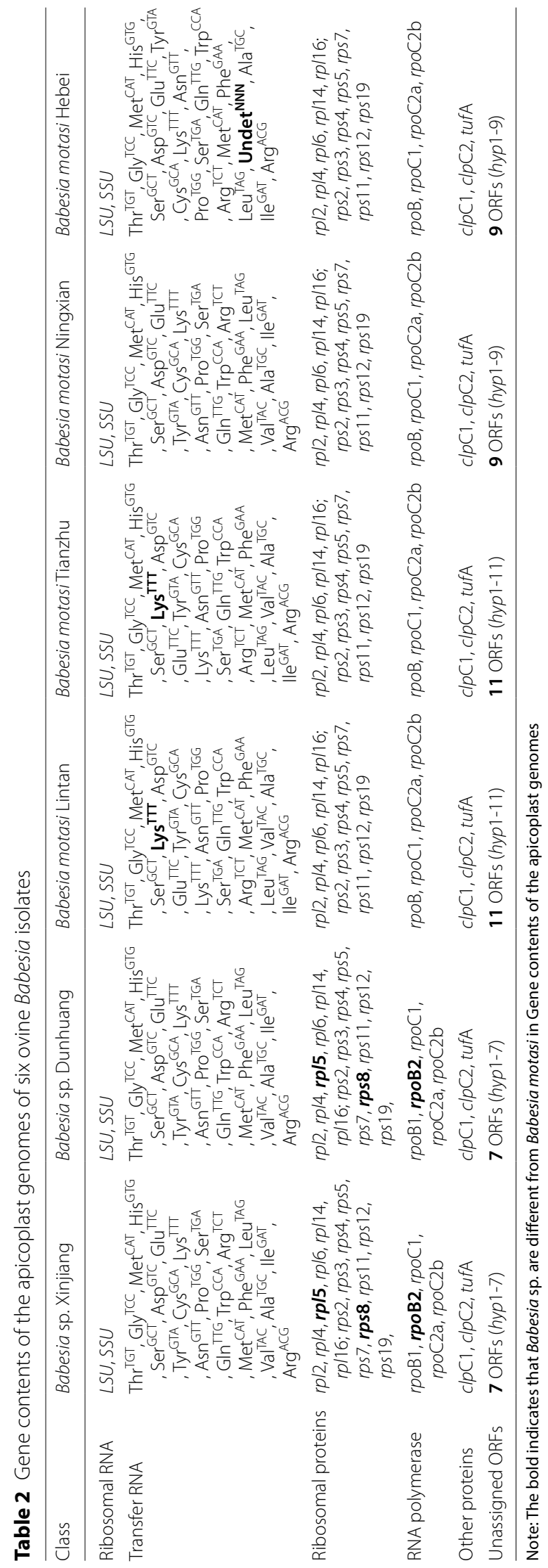


Table 3 Initiation codons and termination codons used in encoding genes of the six ovine Babesia apicoplast genomes

\begin{tabular}{|c|c|c|c|c|c|c|c|c|}
\hline \multirow[t]{2}{*}{ Species } & \multirow{2}{*}{$\begin{array}{l}\text { Total no. of } \\
\text { protein-encoding } \\
\text { genes }\end{array}$} & \multicolumn{4}{|c|}{ Initiation codon } & \multicolumn{3}{|c|}{ Termination codon } \\
\hline & & ATG & ATA & ATT & ATC & TAA & TGA & TAG \\
\hline Babesia sp. Xinjiang ${ }^{\text {a1 }}$ (BspXJ-Sanger, MH992224) & 30 & 30 & 0 & 0 & 0 & 26 & 4 & 0 \\
\hline Babesia sp. Xinjiang ${ }^{\mathrm{a} 2}$ (BspXJ-IIlumina, KX881914) & 30 & 24 & 2 & 3 & 1 & 30 & 0 & 0 \\
\hline Babesia sp. Dunhuang (BspDH, MH992225) & 30 & 30 & 0 & 0 & 0 & 26 & 4 & 0 \\
\hline Babesia motasi Lintan ${ }^{\mathrm{b} 1}$ (BmLT-Sanger, MH992226) & 31 & 31 & 0 & 0 & 0 & 29 & 1 & 1 \\
\hline Babesia motasi Lintan ${ }^{\mathrm{b} 2}$ (BmLT-Illumina, KX881915) & 32 & 30 & 2 & 0 & 0 & 30 & 0 & 2 \\
\hline Babesia motasi Tianzhu (BmTZ, MH992227) & 31 & 31 & 0 & 0 & 0 & 29 & 1 & 1 \\
\hline Babesia motasi Ningxian (BmNX, MH992228) & 29 & 29 & 0 & 0 & 0 & 27 & 1 & 1 \\
\hline Babesia motasi Hebei (BmHB, MH992229) & 29 & 29 & 0 & 0 & 0 & 27 & 1 & 1 \\
\hline
\end{tabular}

a a1 and a2 are the same sample, sequenced using the Sanger and Illumina method, respectively

b b1 and b2 are the same sample, sequenced using the Sanger and Illumina method, respectively

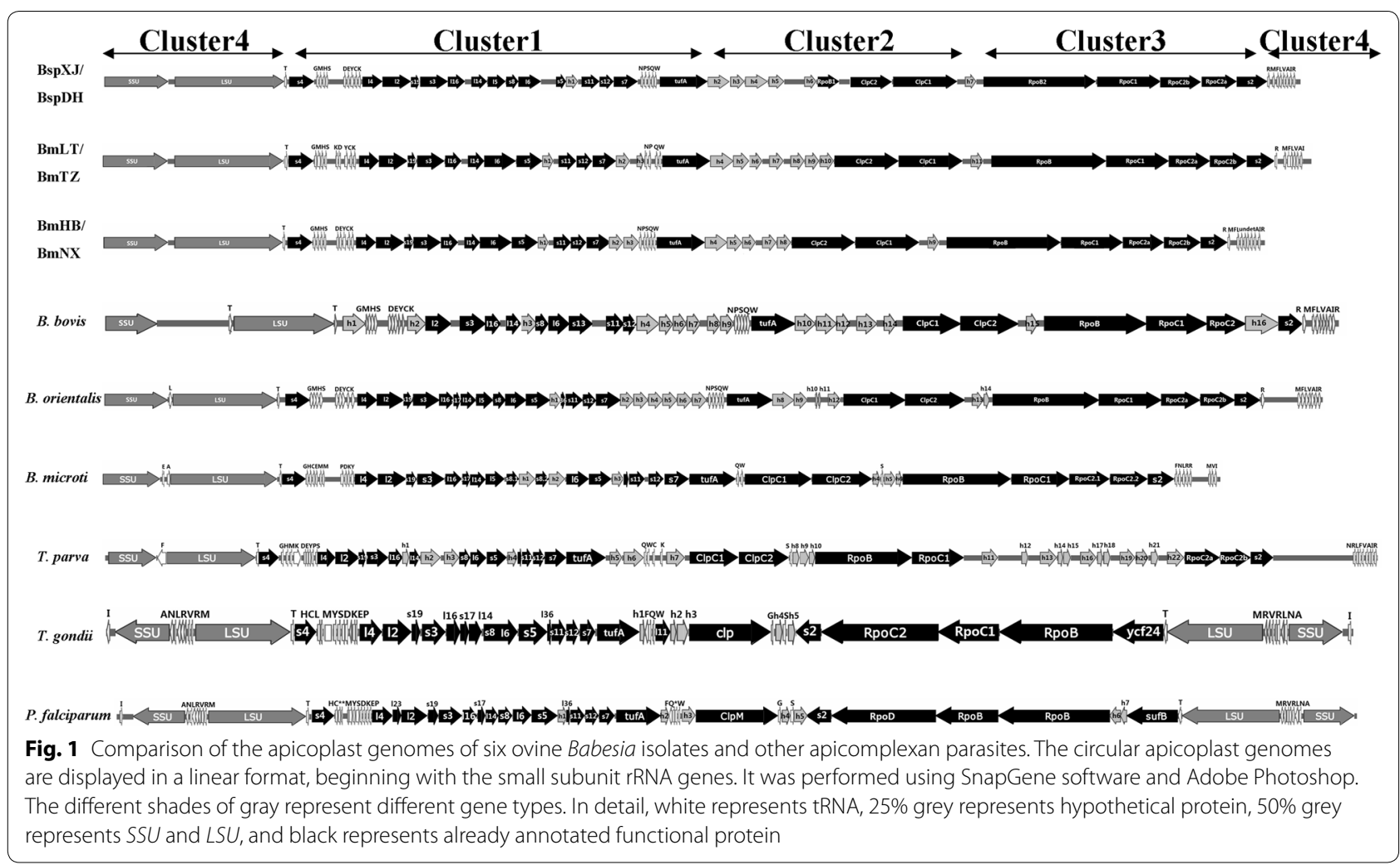

technology (designated BspXJ-Illumina and BmLT-Illumina) were compared with those obtained in this study using the Sanger dideoxy chain-termination method (designated BspXJ-Sanger, MH992224; BmLT-Sanger, MH992226). The results indicated that the size of the apicoplast genome, the number of tRNA and protein-encoding genes, and the initiation and termination codons used in the encoding genes are different in the two sets of data from Sanger and Illumina (Tables 1, 3). In addition, the A
$+\mathrm{T}$ contents and the number of rRNA molecules were consistent but there were differences in the bases at several nucleotide positions along the full-length genome between the first-generation and second-generation data (Table 4).

\section{Phylogenetic analysis}

The ML and NJ trees were constructed using the concatenated amino acid sequences of 13 gene products (4683 


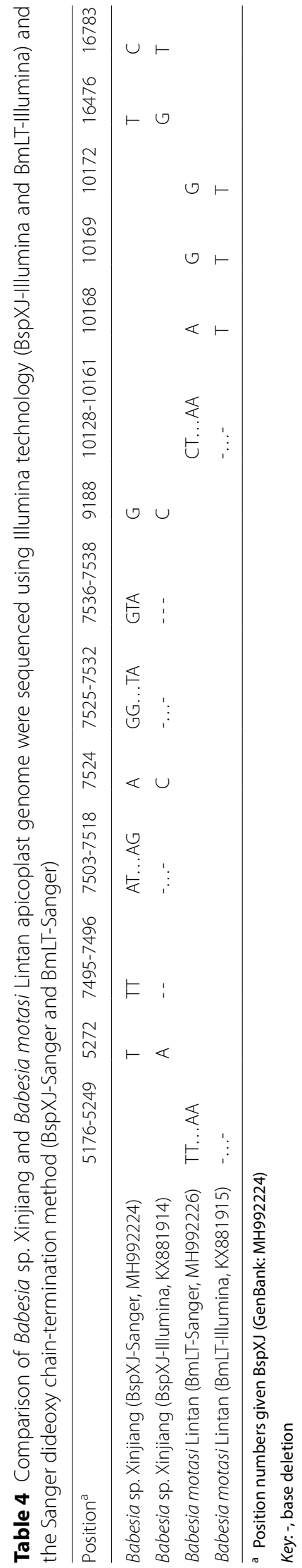


residues) from each apicoplast genomic sequence of 18 apicomplexan parasites and the chloroplast genomic sequence of Chromera velie (as an outgroup). The two approaches gave the same topology. In the amino acid phylogenetic trees, the piroplasms were divided into three groups: classical Babesia infective to ruminants, Theileria and B. microti. The classical Babesia species were separated into three clades: $B$. motasi, Babesia sp. Xinjiang/Dunhuang and B. bovis/B. orientalis. In addition, $B$. motasi were further divided into two subclades: B. motasi Lintan/Tianzhu and B. motasi Ningxian/Hebei (Fig. 2). In the whole apicoplast nucleotide phylogenetic tree, the grouping result for the six ovine Babesia isolates is the same as that of the amino acid phylogenetic trees (Additional file 10: Figure S8).

\section{Discussion}

Babesia spp. are tick-transmitted haemoprotozoans that infect various animal species including humans, causing loss of livestock and public health concern in tropical and subtropical regions of southern Europe, Africa, Asia, Australia and the Americas [9, 36]. The apicoplast is a unique organelle found in apicomplexan parasites; it is considered a target for screening anti-parasitic drugs because it plays important roles in metabolic pathways for fatty acid, iron-sulphur, haem and isoprenoid biosynthesis [43]. In the present study, the apicoplast genomes of six ovine Babesia isolates were sequenced, assembled, annotated and compared with those of other apicomplexan parasites. The apicoplast genomes are smaller than those of most apicoplexan parasites [24, 28, 29, 33, 37, 44], but slightly larger than those of $P$. chabaudi chabaudi [35] and B. microti [25].

The content and order of genes in the cluster differ among the parasites. The rpl23, rpl11 and sufB genes are deficient in the apicoplast genomes of the six ovine Babesia spp., which is similar to $B$. bovis, $B$. orientalis, $B$. microti, T. parva, T. gondii, E. tenella and C. cayetanensis. It is possible that these genes were lost during the genetic evolution of the apicoplast in most apicomplexan parasites [25]. Therefore, some researchers speculate that the rpl 23 and rpl11 genes do not play an important role in the growth and development of these parasites; alternatively, they may be translated at other sites in the apicoplast genome or directly encoded by the nuclear genome. The loss of the sufB gene may have been caused by the

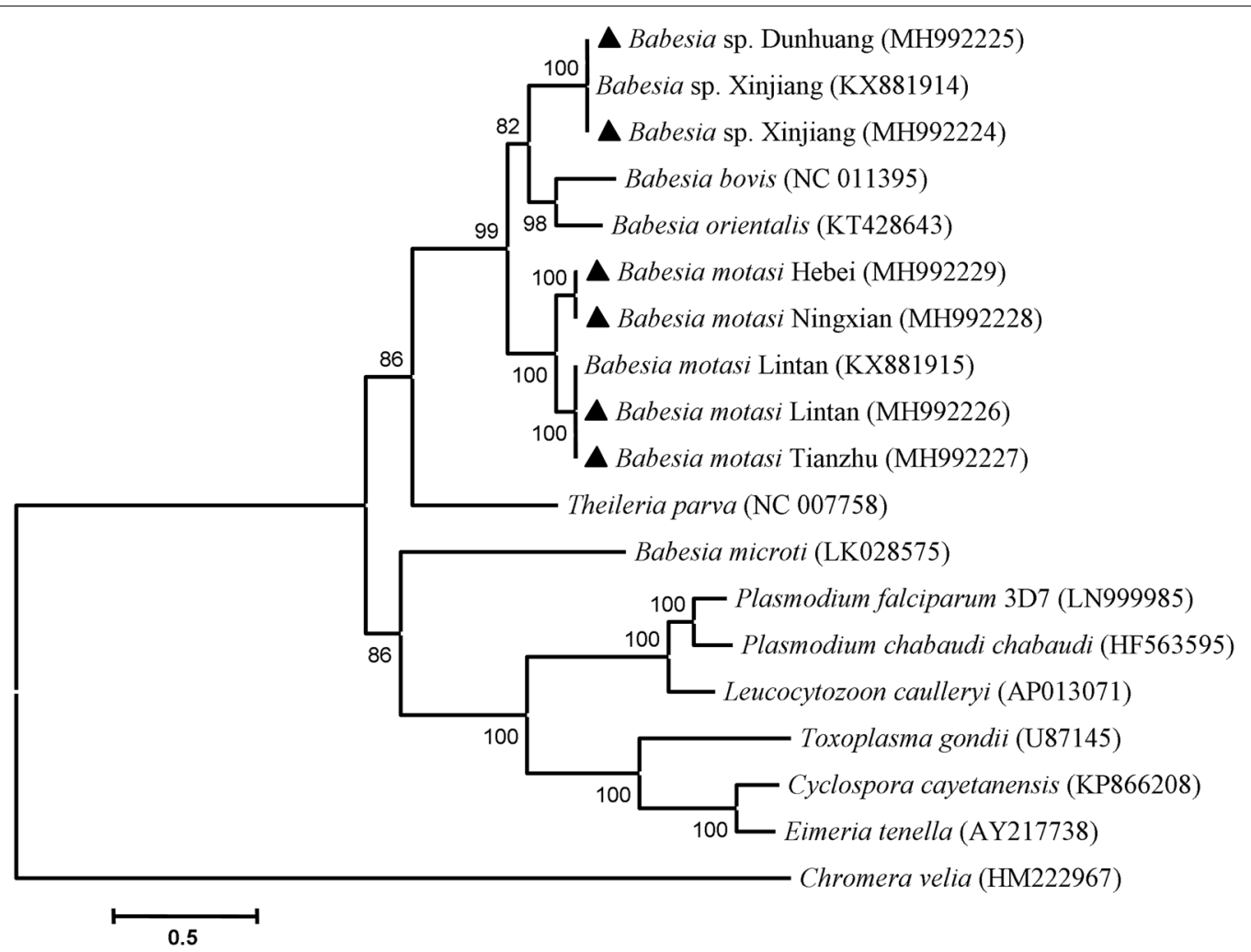

Fig. 2 Phylogenetic relationships of Babesia infective to small ruminants in China and other apicomplexan parasites. Phylogeny was inferred with a maximum likelihood analysis of amino acid sequence data for 13 selected apicoplast genome-encoded genes based on distances calculated with the JTT with Freqs model. Chromera velia (HM222967) was used as the outgroup. Bootstrap values > 50\% from 1000 replicates are shown on the nodes. Babesia obtained in this study is shown as triangles 
gene inversion of cluster 3 during the evolution of Piroplasmida [24, 25]. In this study, different numbers of hypothetical protein genes were found by aligning the apicomplexan apicoplast genomes, which suggests that hyp genes may be one of the causes of the variation in apicoplast genome size. Previous studies have suggested that rearrangements and loss of the genes involved in photosynthesis were thought to be responsible for the formation of apicoplast [24, 25]. Furthermore, gene deletion (sufB, rpl23, rpl11 and hyp), inversion (RNA polymerase), duplication (ClpC and hyp) and restructuring (SSU and $L S U$ ) were also important events during the early evolution of Babesia species. These modifications may have caused the apicoplast genomes of BmNX and $\mathrm{BmHB}$ to be the smallest among the reported Babesia in cattle and small ruminants.

Comparison of the BspXJ and BmLT apicoplast genome data sequenced by first-generation and secondgeneration sequencing techniques showed that there were some differences in genome sizes and the numbers of tRNA and protein-encoding genes. For example, $r p l 5$ and rpl6 genes were present between rpl14 and rps5 genes in the second-generation sequence data of BmLT, while only the rpl6 gene exists in this region in the firstgeneration sequence data of the four $B$. motasi isolates. It is possible that these differences involving nucleotide substitutions, insertions and deletions were due to the different sequencing methods and annotation software used. Additionally, settings of a few parameters comprising ORF length, nested ORFs, start and stop codon, and genetic code were used. Therefore, it is recommended to perform first-generation sequencing to verify sequences based on the second-generation sequencing sequence, to annotate genes using two or more annotation software programs and then further improve the data by using BLAST.

Studies on the biological characteristics (including morphology, pathogenicity, vector tick, serology and in vivo or in vitro propagation) and molecular classification (target genes including SSU, ITS, LSU, HSP90, cox1, cytb, cox3, RPS8 and TRAP) of the Babesia species infective to small ruminants in China have shown that these parasites are divided into two Babesia species: $B$. motasi and Babesia sp. There are two further subspecies of B. motasi, named BmLT/TZ and BmNX/HB (with differences in pathogenicity, serology, in vitro culture features and target gene sequences). These findings are consistent with the Uilenberg inference $[12-15,17,18,20,38,45-51]$. In this study, phylogenetic analyses using concatenated amino acids or whole apicoplast nucleotide sequences confirmed the taxonomical relationships among Babesia species infective to small ruminants in China. In the ML and NJ trees, the ovine Babesia fell into two groups:
Babesia sp. and B. motasi. Babesia motasi was further divided into two small clades, $\mathrm{BmLT} / \mathrm{TZ}$ and $\mathrm{BmHB} / \mathrm{NX}$.

The apicoplast genomes in apicomplexan Babesia parasites remain conserved throughout all the species, including the six ovine Babesia isolates, and Babesia apicoplast functions are significantly different from those of the host [25], suggesting that they might be useful as targets for the development of potent and safe therapies for the treatment of babesiosis. Our data provide useful information confirming the taxonomical relationships of these parasites and identifying targets for anti-apicomplexan parasite drugs.

\section{Conclusions}

In the present study, we have accomplished the sequencing, assembly and annotation of the apicoplast genomes of six ovine Babesia isolates from China. This study has also confirmed our previous inference that there are two Babesia species (Babesia sp. and B. motasi) infective for small ruminants in China, and that the four $B$. motasi isolates possibly belong to two subspecies (BmLT/ $\mathrm{BmTZ}$ and $\mathrm{BmNX} / \mathrm{BmHB})$. Further studies are needed to validate the effects of anti-Babesia drugs against DNA replication, transcription, translation and 2-C-methylD-erythritol 4-phosphate pathways of the apicoplast.

\section{Additional files}

Additional file 1: Table S1. Primers for amplifying apicoplast genomes of six ovine Babesia isolates.

Additional file 2: Figure S1. Circular map of the apicoplast genome of Babesia sp. Xinjiang.

Additional file 3: Figure S2. Circular map of the apicoplast genome of Babesia sp. Dunhuang.

Additional file 4: Figure S3. Circular map of the apicoplast genome of Babesia motasi Lintan.

Additional file 5: Figure S4. Circular map of the apicoplast genome of Babesia motasi Tianzhu.

Additional file 6: Figure S5. Circular map of the apicoplast genome of Babesia motasi Ningxian.

Additional file 7: Figure S6. Circular map of the apicoplast genome of Babesia motasi Hebei.

Additional file 8: Table S2. Comparison of BspXJ/BspDH, BmLT/BmTZ and $\mathrm{BmNX/BmHB}$ apicoplast genomes.

Additional file 9: Figure S7. Whole genome alignment of Babesia obtained in this study with ten genomes from apicomplexan parasites: B. bovis, B. orientalis, B. microti, C. cayetanensis, E. tenella, L. caulleryi, P. chabaudi chabaudi, P. falciparum, T. parva and T. gondii. Comparison was performed using Mauve. The coloured blocks in the first genome are connected by lines to similar blocks in the other genomes. The region of sequence covered by a coloured block is entirely collinear and homologous among the genomes. Each locally collinear block (LCB) is assigned a unique colour and the apicoplast assemblies presented re-arrangements in some species. 
Additional file 10: Figure S8. Phylogenetic relationships of six ovine Babeisa isolates and other apicomplexan parasites. Phylogeny was inferred with a maximum likelihood analysis of whole nucleotide sequences based on distances calculated with the Kimura 2-parameter model. Chromera velia (HM222967) was used as the outgroup. Bootstrap values $>50 \%$ from 1000 replicates are shown on the nodes. Babesia obtained in this study is shown as triangles.

\section{Abbreviations}

DNA: deoxyribonucleic acid; PCR: polymerase chain reaction; ORF: open reading frame; tRNA: transfer ribonucleic acid; rRNA: ribosomal RNA; SSU: small subunit ribosomal RNA; LSU: large subunit ribosomal RNA; rpl: ribosomal protein large subunits gene; rps: ribosomal protein small subunits gene; tufA: elongation factor TU gene; ropB: DNA-directed RNA polymerase subunit beta gene; rpoC: DNA-directed RNA polymerase subunit gene; ClpC: ATP-dependent Clp protease gene; hyp: hypothetical protein; ITS: internal transcribed spacer gene; HSP90: heat-shock protein 90 gene; cox1: cytochrome c oxidase subunit 1 gene; cytb: cytochrome b gene; cox3: cytochrome c oxidase subunit 3 gene; RPS8: ribosomal protein $\$ 8$ gene; RAP-1: rhoptry-associated-protein-1 gene; TRAP: thrombospondin-related anonymous protein gene; Fe:S: ironsulphur; FASII: type II fatty-acid biosynthesis; MEP: 2-C-methyl-D-erythritol 4-phosphate.

\section{Acknowledgements}

Not applicable.

\section{Authors' contributions}

GQG designed and coordinated this study. XXW and GQG carried out the experiments and data analysis and drafted the manuscript. YQL, HY and $J X L$ reviewed the manuscript. JMW, JLL, AHL, XH, JLX, ZL and SYZ extracted the genomic DNA and sequenced the PCR fragments. All authors read and approved the final manuscript.

\section{Funding}

This study was financially supported by the National Key Research and Development Programme of China (2017YFD0501200), 973 Programme (2015CB150300), ASTIP (CAAS-ASTIP-2016-LVRI), NBCIS (CARS-37) and Jiangsu Co-innovation Center Programme for Prevention and Control of Important Animal Infectious Diseases and Zoonoses.

\section{Availability of data and materials}

The datasets generated or analyzed during this study are included in this published article and its additional files. The newly generated sequences were submitted to the GenBank database under the accession numbers MH992224-MH992229.

\section{Ethics approval and consent to participate}

Not applicable.

\section{Consent for publication}

Not applicable.

\section{Competing interests}

The authors declare that they have no competing interests.

\section{Author details}

${ }^{1}$ State Key Laboratory of Veterinary Etiological Biology, Key Laboratory of Veterinary Parasitology of Gansu Province, Lanzhou Veterinary Research Institute, Chinese Academy of Agricultural Science, Xujiaping 1, Lanzhou 730046, Gansu, People's Republic of China. ${ }^{2}$ Jiangsu Co-Innovation Center for the Prevention and Control of Important Animal Infectious Disease and Zoonose, Yangzhou University, Yangzhou 225009, People's Republic of China.

Received: 24 December 2018 Accepted: 19 June 2019

Published online: 24 June 2019

\section{References}

1. Shigeharu S. The apicomplexan plastid and its evolution. Cell Mol Life Sci. 2011:68:1285-96.

2. Zhu G, Marchewka MJ, Keithly JS. Cryptosporidium parvum appears to lack a plastid genome. Microbiology. 2000;146:315-21.

3. Waller RF, Keeling PJ, van Dooren GG, McFadden GL. Comment on "A green algal apicoplast ancestor". Science. 2003;301:49.

4. Fast NM, Kissinger JC, Roos DS, Keeling PJ. Nuclear-encoded, plastidtargeted genes suggest a single common origin for apicomplexan and dinoflagellate plastids. Mol Biol Evol. 2001;18:418-26.

5. Harper JT, Keeling PJ. Nucleus-encoded, plastid-targeted glyceraldehyde3-phosphate dehydrogenase (GAPDH) indicates a single origin for chromalveolate plastids. Mol Biol Evol. 2003;20:1730-5.

6. Lim L, Mcfadden Gl. The evolution, metabolism and functions of the apicoplast. Philos Trans R Soc Lond B Biol Sci. 2010;365:749-63.

7. Sivakumar T, Aboulaila MRA, Khukhuu A, Iseki H, Alhassan A, Yokoyama N, et al. In vitro inhibitory effect of fosmidomycin on the asexual growth of Babesia bovis and Babesia bigemina. J Protozool Res. 2008;18:71-8.

8. Yeh E, Derisi JL. Chemical rescue of malaria parasites lacking an apicoplast defines organelle function in blood-stage Plasmodium falciparum. PLoS Biol. 2011;9:e1001138.

9. Schnittger $L$, Rodriguez AE, Florin-Christensen M, Morrison DA. Babesia: a world emerging. Infect Genet Evol. 2012;12:1788-809.

10. Uilenberg G. Babesia-a historical overview. Vet Parasitol. 2006;138:3-10.

11. Ozubek S, Aktas M. Molecular evidence of a new Babesia sp. in goats. Vet Parasitol. 2017;233:1-8.

12. Guan G, Yin H, Luo J, Lu W, Zhang Q, Gao Y, et al. Transmission of Babesia sp. to sheep with field-collected Haemaphysalis qinghaiensis. Parasitol Res. 2002;88:S22-4.

13. Tian Z, Liu G, Hong Y, Luo J, Guan G, Xie J, et al. Cytochrome c oxidase subunit III (COX3) gene, an informative marker for phylogenetic analysis and differentiation of Babesia species in China. Infect Genet Evol. 2013:18:13-7

14. Tian Z, Luo J, Zheng J, Xie J, Shen H, Yin H, et al. Phylogenetic analysis of Babesia species in China based on cytochrome b (COB) gene. Infect Genet Evol. 2013;13:36-40.

15. Liu AH, Yin H, Guan GQ, Schnittger L, Liu ZJ, Ma ML, et al. At least two genetically distinct large Babesia species infective to sheep and goats in China. Vet Parasitol. 2007;147:246-51.

16. Niu Q, Luo J, Guan G, Liu Z, Ma M, Liu A, et al. Differentiation of two ovine Babesia based on the ribosomal DNA internal transcribed spacer (ITS) sequences. Exp Parasitol. 2009:121:64-8.

17. Guan G, Moreau E, Liu J, Hao X, Ma M, Luo J, et al. Babesia sp. BQ1 (Lintan): molecular evidence of experimental transmission to sheep by Haemaphysalis qinghaiensis and Haemaphysalis longicornis. Parasitol Int. 2010:59:265-7.

18. Guan G, Ma M, Moreau E, Liu J, Lu B, Bai Q, et al. A new ovine Babesia species transmitted by Hyalomma anatolicum anatolicum. Exp Parasitol. 2009:122:261-7.

19. Guan G, Ma M, Liu A, Ren Q, Wang J, Yang J, et al. A recently identified ovine Babesia in China: serology and sero-epidemiology. Parasitol Int. 2012;61:532-7.

20. Bai Q, Liu G, Liu D, Ren J, Li X. Isolation and preliminary characterization of a large Babesia sp from sheep and goats in the eastern part of Gansu Province, China. Parasitol Res. 2002;88:S16-21.

21. Niu Q, Liu Z, Yang J, Yu P, Pan Y, Zhai B, et al. Expression of sheep pathogen Babesia sp. Xinjiang rhoptry-associated protein 1 and evaluation of its diagnostic potential by enzyme-linked immunosorbent assay. Parasitology. 2016;143:1990-9.

22. Niu Q, Liu Z, Yang J, Yu P, Pan Y, Zhai B, et al. Expression analysis and biological characterization of Babesia sp. BQ1 (Lintan) (Babesia motasi-like) rhoptry-associated protein 1 and its potential use in serodiagnosis via ELISA. Parasit Vectors. 2016;9:313.

23. Wang JM, Ma ML, Liu AH, Ren OY, Li AY, Liu ZJ, et al. A sero-epidemiological survey of Chinese Babesia motasi for small ruminants in China. Parasitol Res. 2013;112:2387-91. 
24. Huang Y, He L, Hu J, He P, He J, Yu L, et al. Characterization and annotation of Babesia orientalis apicoplast genome. Parasit Vectors. 2015;8:543.

25. Garg A, Stein A, Zhao W, Dwivedi A, Frutos R, Cornillot E, et al. Sequence and annotation of the apicoplast genome of the human pathogen Babesia microti. PLoS ONE. 2014;9:e107939.

26. Williamson DH, Denny PW, Moore PW, Sato S, Mccready S, Wilson RJ. The in vivo conformation of the plastid DNA of Toxoplasma gondii: implications for replication. J Mol Biol. 2001;306:159-68.

27. Lau AOT, Mcelwain TF, Brayton KA, Knowles DP, Roalson EH. Babesia bovis: a comprehensive phylogenetic analysis of plastid-encoded genes supports green algal origin of apicoplasts. Exp Parasitol. 2009;123:236-43.

28. Brayton KA, Lau AOT, Herndon DR, Hannick L, Kappmeyer LS, Berens SJ, et al. Genome sequence of Babesia bovis and comparative analysis of apicomplexan hemoprotozoa. PLoS Pathog. 2007;3:1401-13.

29. Cai X, Fuller AL, McDougald LR, Zhu G. Apicoplast genome of the coccidian Eimeria tenella. Gene. 2003;321:39-46.

30. Cinar HN, Qvarnstrom Y, Weipridgeon Y, Wen L, Nascimento FS, Arrowood MJ, et al. Comparative sequence analysis of Cyclospora cayetanensis apicoplast genomes originating from diverse geographical regions. Parasit Vectors. 2016;9:611

31. Wilson RJ, Denny PW, Preiser PR, Rangachari K, Roberts K, Roy A, et al. Complete gene map of the plastid-like DNA of the malaria parasite Plasmodium falciparum. J Mol Biol. 1996;261:155-72.

32. Saxena V, Garg S, Tripathi J, Sharma S, Pakalapati D, Subudhi AK, et al. Plasmodium vivax apicoplast genome: a comparative analysis of major genes from Indian field isolates. Acta Trop. 2012;122:138-49.

33. Imura T, Sato S, Sato Y, Sakamoto D, Isobe T, Murata K, et al. The apicoplast genome of Leucocytozoon caulleryi, a pathogenic apicomplexan parasite of the chicken. Parasitol Res. 2014;113:823-8.

34. Arisue N, Hashimoto T, Mitsui H, Palacpac NM, Kaneko A, Kawai S, et al. The Plasmodium apicoplast genome: conserved structure and close relationship of $P$. ovale to rodent malaria parasites. Mol Biol Evol. 2012;29:2095-9.

35. Sato S, Sesay AK, Holder AA. The unique structure of the apicoplast genome of the rodent malaria parasite Plasmodium chabaudi chabaudi. PLOS ONE. 2013;8:e61778.

36. Tao W, Guan G, Korhonen PK, Koehler AV, Hall RS, Young ND, et al. The apicoplast genomes of two taxonomic units of Babesia from sheep. Vet Parasitol. 2016;233:123-8.

37. Gardner MJ, Bishop R, Shah T, de Villiers EP, Carlton JM, Hall N, et al. Genome sequence of Theileria parva, a bovine pathogen that transforms lymphocytes. Science. 2005;309:134-7.

38. He X, Liu JL, Liu AH, Wang JM, Niu QL, Li YQ, et al. The structural and phylogenetic analysis of trap gene in ovine Babesia species in China. Acta Vet Zootech Sin. 2017;48:1332-41.
39. Carver T, Harris SR, Berriman M, Parkhill J, Mcquillan JA. Artemis: an integrated platform for visualization and analysis of high-throughput sequence-based experimental data. Bioinformatics. 2012;28:464-9.

40. Lowe TM, Chan PP. tRNAscan-SE On-line: integrating search and context for analysis of transfer RNA genes. Nucleic Acids Res. 2016;44:W54-7.

41. Grant JR, Stothard P. The CGView Server: a comparative genomics tool for circular genomes. Nucleic Acids Res. 2008;36:W181-4.

42. Darling AE, Mau B, Perna NT. progressiveMauve: multiple genome alignment with gene gain, loss and rearrangement. PLOS ONE. 2010:5:e11147.

43. McFadden Gl. The apicoplast. Protoplasma. 2011;248:641-50.

44. Tang K, Guo Y, Zhang L, Rowe LA, Roellig DM, Frace MA, et al. Genetic similarities between Cyclospora cayetanensis and cecum-infecting avian Eimeria spp. in apicoplast and mitochondrial genomes. Parasit Vectors. 2015;8:358.

45. Wang T, Guan G, Korhonen PK, Koehler AV, Young ND, Hall RS, et al. Mitochondrial genomes of two Babesia taxa from sheep in China as a foundation for population genetic and epidemiological investigations. Infect Genet Evol. 2017;47:51-5.

46. Tian ZC, Liu GY, Yin H, Luo JX, Guan GQ, Luo JX, et al. RPS8 - a new informative DNA marker for phylogeny of Babesia and Theileria parasites in China. PLoS ONE. 2013;8:e79860.

47. Liu GY, Lian S, Han GF, Wang ZD, Wang MY, He HC, et al. Transmission test of two ovine Babesia in Ningxian county, Gansu Province. Ch Vet Sci. 1997:27:10-1.

48. Ma ML, Guan GQ, Gou HT, Yang JF, Wan YL, Li YQ, et al. Discovery of an ovine Babesia sp. strain from sheep in Dunhuang city of Gansu Province. Ch Vet Sci. 2011:41:881-5.

49. Lian C, He HC, Bai Q, Han GF, Liu GY. A large Babesia sp. new-found from affected sheep in China. Ch Vet Sci. 1997;17:116-9.

50. Guan G, Liu J, Liu A, Li Y, Niu Q, Gao J, et al. A member of the HSP9o family from ovine Babesia in China: molecular characterization, phylogenetic analysis and antigenicity. Parasitology. 2015;142:1387-97.

51. Gou HT, Guan GQ, Ma ML, Liu AH, Liu ZJ, Ren QY, et al. Phylogenetic analysis based on 28S rRNA of Babesia spp. in ruminants in China. Exp Appl Acarol. 2013;59:463-72.

\section{Publisher's Note}

Springer Nature remains neutral with regard to jurisdictional claims in published maps and institutional affiliations.
Ready to submit your research? Choose BMC and benefit from:

- fast, convenient online submission

- thorough peer review by experienced researchers in your field

- rapid publication on acceptance

- support for research data, including large and complex data types

- gold Open Access which fosters wider collaboration and increased citations

- maximum visibility for your research: over 100M website views per year

At BMC, research is always in progress.

Learn more biomedcentral.com/submissions 